\title{
Health consequences of bulimia nervosa
}

\author{
Walter Milano, Luca Milano and Anna Capasso* \\ Department of Pharmacy, University of Salerno, Salerno, Italy
}

\begin{abstract}
Bulimia Nervosa (BN) is coded for the presence of binge eating (binge eating) depression, characterized by the intake of a quantity of food that is significantly greater than that of most people (for example, two hours) would eat at the same time and in the same circumstances with a subjective feeling of loss of control over what you eat during the episode (for example, the feeling that you cannot stop eating or not being able to control what and how much you eat), followed by compensatory behaviors designed to minimize weight gain such as self-induced vomiting, misuse of laxatives and/or diuretics, fasting, intense physical activity, and clisters. BN can be difficult to identify because of the experience of secrecy and shame where the patient segregates binge crises and compensatory behaviors. The weight may be normal or slightly higher than normal. Often, in history, there is a history of AN or restrictive diet

The health consequences of $\mathrm{BN}$ are extremely variable and can occur with only modest biological and physical damage up to extremely serious and life-threatening conditions; the mortality rate of young subjects. The management of the medical-internship aspects of BN is rightly placed within complex and articulated programs of interdisciplinary treatment with different levels of intensity of care (outpatient, semi-residential/residential, hospital in cases of emergency/medical and/or psychiatric emergency).
\end{abstract}

This review focuses on health consequences associated with bulimia nervosa.

\section{Introduction}

Bulimia nervosa (BN) defines clinical patterns characterized by aberrations of eating behavior, with potentially serious clinical repercussions.

The bulimic patient alternates between restrictive periods and periods with hyperphagic access followed by vomiting; abuse of laxatives, diuretics and slimming drugs; often an exhausting physical activity is the first symptom of an excessive desire for weight reduction [1-3].

The $\mathrm{BN}$ is characterized by a wide spectrum of clinical presentations: from the mildest form that can be spontaneously reversed, to the severe forms that undergo progressive deterioration. In over a third of cases, difficulties in social and family adaptation persist despite clinical recovery [1-3].

Hospitalization becomes necessary when the patient has severe bulimic episodes, depressive crises with suicidal intent or important metabolic imbalances. In addition to suicides, general mortality is linked to the complications of malnutrition, including infectious episodes, cardiovascular collapse, electrolyte imbalances and finally cardiac arrhythmias [1-3].

Early expression of bulimia nervosa is the damage of the digestive system, from the oral cavity to the intestine. The symptomatology is quite variable and is often hidden to the doctor: it becomes therefore an unavoidable task of the doctors involved in the diagnosis and coordinated care of the bulimia nervosa to decode as soon as possible the most strictly clinical symptoms. It is important to remember that the bulimic patient is usually normal or overweight $[4,5]$.

\section{Dental complications}

Bulimia nervosa can lead to a series of odontostomatological manifestations (which may involve hard tissues, soft tissues and salivary glands) classified in primary and secondary. Among the primaries we remember dental erosions up to exposure of the pulp, loss of substance or fracture of teeth, increased incidence of caries, dentinal hypersensitivity, xerostomia, periodontal diseases, glossodynia, cheilitis. Among the secondary ones there is the hypertrophy of the salivary glands, especially of the parotid, the occlusal anomalies, the impairment of the masticatory capacity, the aesthetic alterations [6].

Dental erosions are related to self-induced vomiting and gastroesophageal reflux, due to the acidity of gastric juice [6]. The carious pathology seems to be due to a decrease in salivary $\mathrm{pH}$ typical of post-vomiting, with consequent bacterial proliferation and abatement of the buffering power of saliva, as well as the type of diet, rich in carbohydrates and sugary foods (candies, chocolate, etc.) [7]. In many cases we can observe a parotid hypertrophy, related to malnutrition, chronic glandular stimulation reflected by excessive carbohydrate intake, buccal mucosal irritation by self-induction of vomiting or alcohol abuse [8].

It can be said that all the dental complications stated are related to vomiting, to the reduction of salivary flow, to gastroesophageal reflux and can be progressive and serious. Since they already occur early in the disease, clinicians who take care of ED should be urged to also include the dentist in the staff, in order to monitor, prevent and treat the injuries present.

${ }^{\star}$ Correspondence to: Anna Capasso, Department of Pharmacy, University of Salerno, Salerno, Italy, Tel: (304) 293-5110; E-mail: annacap@unisa.it

Key words: bulimia nervosa, eating disorders, health consequences, psychiatric emergency

Received: February 11, 2018; Accepted: February 23, 2018; Published: February 27,2018 


\section{Digestive system complications}

In bulimic patients, gastrointestinal tract disorders may be the early expression of the disease and the symptoms related to this district may represent the most evident clinical problem in subjects with still misunderstood forms of eating disorders (ED). For these reasons, these patients often turn to the gastroenterologist first, who with symptomatic care controls the clinical picture, but delaying the diagnosis of years [9].

Once the diagnosis of $\mathrm{BN}$ has been established and effective treatment has been undertaken, however, many of the alterations in the digestive tract are reduced or disappear. In some cases, however, especially when the bulimia has lasted for many years and the clinical control of the condition has not been satisfactory for long periods, symptoms and alterations related to the digestive system, such as disorders of gastric emptying or colon motility, they can persist even in the phases of effective control of the disease. In these cases, the persistence of dyspeptic symptoms can facilitate relapse towards altered eating behaviors. It is important for these reasons to recognize in a young patient with gastrointestinal symptoms the stigmata of the $\mathrm{ED}$, as well as for the psychiatrist the conditions that require medical treatment: the multidisciplinary management must be proper to this affection [9].

In general, the medical complications of bulimia are mainly due to functional and metabolic alterations of binge episodes and compensatory behaviors (self-induced vomiting, laxative abuse). In particular, gastrointestinal ones can be classified by district [9].

At the level of the oropharynx we remember, in addition to the dental ones and the already described parotid hypertrophy, lesions of the oropharyngeal and lingual mucosa, related to acid regurgitation, hyperphage, and vomiting [9].

The esophagus may have lesions ranging from mild erosive esophagitis to rupture of the esophagus. Esophagitis, erosions, esophageal ulcers, bleeding are the result of the mucosal insult of chloridro-peptic material during repeated episodes of self-induced vomiting. If this behavior persists, it is possible to develop cicatricial esophageal strictures with dysphagia or in Barret's esophagus. In some patients the continuous esophageal trauma causes reduced lower esophageal sphincter tone and this condition promotes the perpetuation of acid gastroesophageal reflux, complicating the picture [9]. The esophageal rupture, on the other hand, is very rare and consists of tearing of the esophageal wall, usually in the distal third, due to barotrauma following induced vomiting of an imposing volume of food taken during a binge episode (Boerhaave's). This is a severe clinical condition, which can be complicated by mediastinitis and lead to death of the patient and is characterized by vomiting, retrosternal pain, subcutaneous emphysema. Esophageal laceration with hematemesis, described by Mallory-Weiss in alcoholics following repeated vomiting, may occur, albeit rarely, even in patients with bulimia nervosa, requiring intensive treatment [9].

The stomach is mainly involved in the genesis of symptoms in bulimia nervosa is certainly central and from the first observations were described bulimic patients arrived at the observation of doctors and surgeons for important gastrectasis. Gastric emptying usually appears to be slowed down [10], but in some cases it is also described as accelerated: a large part of gastric symptoms seems to be due to motor alterations, mainly due to altered antral motility. Quite rare is the occurrence, in bulimics after binge, of the acute over dissension of the stomach, with possible rupture of the gastric wall [11]. This complication could be caused not only by the abrupt mechanical relaxation of the walls but also by the reduced resistance due to malnutrition and the reduced contractility resulting from electrolyte disturbances (hypokalemia). Sometimes the duodenum is also involved in the dilatation of the stomach with duodenal ileus, causing in turn obstruction to the biliary and pancreatic outflow, evidenced by the rise in the indices of colostasis and pancreatic enzymes. Recently, it has also been shown that in patients with $\mathrm{BN}$, meals, even if of large volume, cause a modest increase in cholecystokinin (CCK) compared to normal subjects [12]. This hormone normally causes a feeling of satiety that tends to curb the intake of other food; in bulimics, however, this negative feedback mechanism is reduced, facilitating the tendency to compulsive binge. Ultimately, it is likely that the alterations of gastric motility play an important role in the clinical history of $\mathrm{BN}$, but that they are only secondary to behavioral disorders. The main problem seems to be not how the stomach moves, but how the patient perceives the signs of hunger and satiety coming from the first part of the alimentary canal [12].

In bulimic patient's motility disorders are also at the intestinal level. The main alteration is clinically expressed with transit disorders and constipation. A form of high intestinal obstruction is represented by the syndrome of the superior mesenteric artery (SMAS), however rare in bulimia nervosa $[5,12]$.

Constipation is quite common in the $\mathrm{BN}$; the slowing seems mainly due to a slow emptying of the colon rather than a slowed transit in the small intestine. The rectal distention caused by the hypomotility of the colon causes, in turn, inhibition of gastric emptying, further interfering with transit times, but also on the sense of hunger and satiety. Furthermore, the subjective sensation of abdominal sweating and swelling can exacerbate the patient's fear of gaining weight. Do not forget the effects of the abuse of laxatives, treated subsequently, which can cause excessive loss of liquids and ions (potassium), stimulation of the plexus of Auerbach and therefore of the peristalsis of the colon with degeneration of intestinal innervation and consequent irreversible colon hypotonia, to configure the framework of severe colonic inertia of the so-called colon from cathartics $[5,12]$.

As for the stomach, visceral sensitivity plays a role at least equal to that of motility, even for the intestine. In turn, the perception of symptoms from the alimentary tract is closely linked to the psyche and the mechanisms that regulate behavior and mood. In bulimic patients treated with antidepressive therapy, the gastrointestinal symptom score related to meals is reduced, calculated on the frequency and intensity of symptoms such as dyspepsia, abdominal pain, constipation, frequent belch, nausea, heartburn, flatulence and bowel sounds $[5,12]$.

To conclude, we must not forget that gastrointestinal disorders can often divert the doctor from the right diagnosis, risking settling for an irritable colon for a bulimic patient or the opposite risk of ED for a chronic intestinal inflammatory disease. The better knowledge of the mechanisms of the $\mathrm{BN}$ and the best collaboration between specialists can avoid dangerous delays in diagnosis and incongruous treatments, with a greater possibility to deal with this complex pathological situation promptly and effectively $[5,12]$.

\section{Hepatic and pancreatic complications}

During the course of the BN, the mechanisms of elimination and malnutrition also cause hepatic and pancreatic changes. The liver, in the process of restriction, undergoes a steatosis more or less severe that rarely evolves into cirrhosis and which appears reversible after 
re-feeding. Hepatic suffering also manifests with fluctuations of some liver enzymes: with an increase in transaminases, LDH, gamma-GT, alkaline phosphatase; with reduction of cholinesterase and plasma proteins [12]. Malnutrition also causes morphological and functional pancreatic complications. Hyperamylasemia is often due to an increase in amylase of salivary origin, linked to an increase during the frequent bulimic crises with a high content of carbohydrates: in this case there is also marked hypertrophy of the salivary glands [13]. To ascertain pancreatic involvement, it is necessary to determine the plasma values of isoamylases and pancreatic lipase whose modifications accompany the variations of pancreatic amylases. If the lipase values are normal, hyperamylasemia may presumably not have a pancreatic origin and it is not necessary to perform diagnostic tests. Cases of acute pancreatitis have also been described, but the determining mechanism has not yet been clarified, they are probably linked to problems of difficult Wilsung's duct outflow [14].

\section{Metabolic and electrolytic complications}

In the $\mathrm{BN}$, metabolic imbalances are frequent, in fact, in malnutrition, the need for glucose and essential amino acids is assured by the reserves of hepatic glycogen and therefore by the catabolism of adipose and muscular tissue. The preferential use of adipose tissue is responsible for ketosis and ketonuria. The latter, by inhibiting the tubular secretion of uric acid, which is also associated with the abuse of diuretics, facilitates the occurrence of hyperuricemia [15]. Regarding the hydroelectrolytic imbalances, even if the values of potassium are not always reduced, the total body potassium has decreased, as a consequence of the repeated episodes of vomiting and the abuse of diuretics and cathartics. Loss of acids with vomiting causes hypochloremic and hypokalemic metabolic alkalosis with increased $\mathrm{pCO}_{2}$. Hypokalaemia, in turn, promotes further renal loss of acids, while the use of diuretics and laxatives worsens hypochloraemia. Malnutrition causes a decrease in the total body content of sodium; diarrhea and vomiting on the one hand and diuretics on the other, cause a loss of magnesium that can worsen hypokalemia and dilute calcium levels. Electrolyte imbalance can naturally cause severe disturbances of the heart rhythm and, associated with metabolic alterations, precipitate a decompensation [16]. These electrolyte alterations are particularly dangerous as they can be fleeting and therefore not be recognized in good times; already 24-48 hours after an episode of vomiting, in fact, potassium and chloride return to normal values. Recently, attention has been paid to the possible pathogenic role of zinc deficiency in some clinical manifestations of malnutrition. In fact, zinc deficiency induces weight loss, growth retardation and sexual development, changes in mood, ageusia, decreased appetite and skin changes and appendages. Zinc deficiency can result from inadequate diuretics, altered intestinal absorption, loss of sweat in hyperactive patients and increased urinary elimination in bulimics who abuse alcohol [17].

\section{Cardiovascular complications}

In recent years the prevalence and incidence of $\mathrm{BN}$ are progressively increasing with the involvement of the younger age groups, with a worrying increase in mortality in this age group, mainly related to cardiovascular complications. These considerations show the need and interest in focusing attention on the cardiovascular changes of bulimic patients [18-20].

The neurosensory changes typical of malnutrition are reflected on the cardiovascular system with an adaptation, only initially with homeostatic value, which results in alterations of the cellular pathophysiology of the myocardiocytes and of the endothelium and therefore, globally, of the hemodynamics. The reduction of the cardiac index takes second place to the decrease of the systolic range and of the heart rate. Because of the negative isotropic action and chronotropic incompetence, always linked to malnutrition, we can notice an early fatigue. The impaired ability to ensure an adequate increase in heart rate in response to physical activity depends mainly on changes in the autonomic nervous system (ANS), which may in some cases induce hypotension. Electrocardiographic alterations are also possible, such as low voltage, QT time extension, ST tract depression, T-wave inversion and atrial and ventricular premature beats. The $U$ wave and the prolongation of the QT time, with an increased risk of tachyarrhythmias, prevail in the bulimics, in which hypokalaemia and hypomagnesemia are more pronounced [21].

\section{Dermatological complications}

The correlation of many dermatoses with BN is still the subject of discussion, at least to our knowledge there are no studies on extensive cases concerning early cutaneous manifestations or the variation of cutaneous signs in relation to the course of this affection. Among the cutaneous manifestations, however, some may be considered nonspecific, other specific: the latter are pathognomonic and allow to suspect an allergic contact dermatitis in the absence of other clinical signs. In the BN the most obvious aspects may be skin dryness and the sign of Russell, that is calluses on the back of the hands caused by the introduction of the fingers in the throat that rub against the incisors, to obtain vomiting. The cutaneous manifestations can be classified according to the four main causal factors: malnutrition; self-induced vomiting; improper use/abuse of laxatives, diuretics and emetics; concomitant psychiatric disorders [22,23]. Those from malnutrition are the most frequent include xerosis and asteatosic dermatitis, acne, carotenoderma, irritative dermatitis, seborrheic dermatitis and striae distaense [24]. Malnutrition is also due to the fragility of the nails and hair and the dystrophic appearance of the skin that appears pale yellowish-yellow, dry, cold, apparently dirty and slightly desquamating. Among the complications related to self-developed vomiting we mention erosions, pigmented scars and above all the circumscribed calluses of the back of the hands. The calluses of the back of the hands, known as the sign of Russell, represent, in fact, a guiding diagnostic sign for the diagnosis of ED when other clinical signs are not yet detectable. The manifestations of use/abuse of laxatives, diuretics, and emetics include adverse reactions from these drugs, such as purpura, photosensitivity reactions, urticaria, pruritus, exfoliative dermatitis, tonic acid, polymorphic erythema and cutaneous vasculitis. Finally, from concomitant psychiatric pathologies are the artifact dermatoses, which coexist more often with anorexia nervosa than with bulimia nervosa and are self-persecuted lesions of bizarre shape, located in regions easily reachable by the hands, such as hand and forearm left, legs and feet (24).

\section{Infectious complications}

Although poor literature does not provide a clear interpretation of the incidence of infections in bulimia nervosa, patients with this disorder may suffer respiratory infections, especially at ab ingestis pneumonias, since repeated vomiting is a predisposing factor for this pathology [25]. Other factors that promote the aspiration of gastric contents in the respiratory system are gastroesophageal reflux, dysphagia, improper intake of psychoactive drugs, and the use of devices that alter pharyngeal motility, such as the positioning of the nasogastric tube. From a clinical point of view there are three types of ab ingestis pneumonia: chemical, bacterial and mechanical obstruction. Chemical 
pneumonia is caused by the penetration of toxic substances into the airways that stimulate an inflammatory reaction regardless of the bacterial load and bronchial obstruction. The clinical features include dyspnoea, cyanosis and bronchospasm that arise acutely and resolve within 4-7 days without reliquaries. In extreme cases, hyaline membranes syndrome or adult respiratory distress syndrome (ARDS) may occur [25]. Bacterial pneumonia is instead caused by aspiration and on the clinical level is characterized by the fact that the classic hallmarks of pneumonia (fever, cough, chest pain) may be missing. This is due to the poor general conditions of the host that determine energy to the infectious process and cause attenuation or total lack of constitutional symptoms $[5,12]$. Added to this is that many patients tend to deny or minimize their discomfort, so it follows that sometimes the diagnosis can be delayed, and the pneumonia can evolve towards narcotization, abscess or empyema. The responsible bacterial flora is mixed: the anaerobic bacteria are always involved in association, but in a discrete percentage of cases also aerobic pathogens are isolated. Gram negative bacteria are implicated in patients in whom the gastric contents are polluted by the enteric flora and their presence is associated with higher mortality [5,12]. Finally, pneumonia from mechanical obstruction of the airways occurs when small solids cause total or partial obstruction of a bronchus for more than a week, which allows the proliferation of anaerobic bacterial flora. Finally, clinical and epidemiological data indicate, in bulimic patients, obesity as a risk factor for surgical infections [26]. In particular, some authoritative studies have shown that the risk of postoperative wound infection is higher in severely overweight patients compared to normal BMI subjects, associating this risk with antibiotic doses administered to these patients, which are inadequate in relation to their greater body weight.

\section{Effects of laxative abuse}

Patients who have problems with compulsive binge eating use laxatives to influence physical fitness and weight, although this practice is less common than self-induced vomiting. Laxative abuse can be responsible for complications in the acid-base and electrolyte balance: a severe acute diarrhea determines a condition of hyperchloremic metabolic acidosis, while chronic diarrhea from repeated abuse of laxatives can cause a metabolic, hypochloremic and alkalosis hypokalemia, exacerbated by the excess of mineralocorticoids linked to hypovolemia [27]. The chronic abuse of these drugs can also cause dependence pictures with loss of the physiological peristaltic activity of the colon following the stimulation of the plexus of Auerbach and subsequent degeneration of intestinal innervation. In this way, the picture of severe colonic inertia of the so-called catartic colon can be seen, highlighted with RX opaque clisma: the colon appears dilated and atonic and microscopically thinning microvilli and alterations in the cytoplasmic orphans, lysosomes and nerve plexuses are observed at the microscopic level. Laxative suspension can be associated with reflex constipation, fluid retention and abdominal swelling sensation, which can cause the patient to resume the excessive intake of food. The manifestations of use/abuse of laxatives include, finally, also the adverse reactions deriving from these drugs which (as already described in dermatological complications) are mainly represented by the purple, photosensitivity reactions, urticaria, pruritus, exfoliative dermatitis, tossidermie, erythema polymorphic and cutaneous vasculitis [27].

\section{Conclusions}

The main health consequences of bulimic subjects are injury to the gastrointestinal tract, skin problems, hair loss, increased serum amylase levels, metabolic acidosis and hormonal imbalances that lead to amenorrhea in women (lack of menstrual cycle), up to electrolyte imbalance (hypokalemia, hyponatraemia, hypochloraemia); a severe lack of potassium and sodium due to compensatory mechanisms, which can lead to cardiac arrhythmias and even death. In the rare cases of bulimia secondary to other pathology, the therapy obviously consists in the treatment of the underlying disease. The therapy of bulimia nervosa, on the other hand, can be both psychological and pharmacological, possibly in association [28-30]. The psychological approach employs qualified medical personnel and can provide individual, family or group psychotherapy sessions; the latter seem to be the most effective, especially in adolescents and young women, but also in young male subjects. Health facilities offer, generally for free, the possibility to participate in self-help groups. The most followed therapeutic model is that of cognitive-behavioral therapy (TCC) [4], in many cases associated with dietary advice and pharmacological treatments based on antidepressant drugs [28-30]. Pharmacological treatments generally consist, as mentioned above, in the use of antidepressant drugs belonging to the category of SSRIs (selective serotonin reuptake inhibitors); the intake of these drugs has shown a certain effectiveness in the treatment of bulimia nervosa; firstly because it is generally observed, within a few weeks, a reduction in the frequency of binges of about $50 \%$ and secondly there is a reduction of about $50 \%$ in episodes of vomiting with consequent improvement of the tone of mood and sense of control over nutrition [28-30].

\section{References}

1. Nicholls D, Bryan-Waugh R (2008). Eating disorders of infancy and childhood: definition, symptomatology, epidemiology, and comorbidity. Child Adolesc Psychiatr Clin N Am 18: 17-30. [Crossref]

2. AED (2011) Eating disorders, critical points for early recognition and medical risk management in the care of individuals with eating disorders. Report, $\left(2^{\text {nd }}\right.$ edn) Academy for Eating Disorders.

3. Volpe U, Tortorella A, Manchia, Monteleone AM, Albert U, et al. (2016) Eating disorders: What age at onset? Psychiatry Res 238: 225-227. [Crossref]

4. Hail L, Le Grange D (2018) Bulimia nervosa in adolescents: prevalence and treatment challenges. Adolesc Health Med Ther 9: 11-16. [Crossref]

5. Mehler PS, Rylander M (2015) Bulimia Nervosa - medical complications. J Eat Disord 3: 12. [Crossref]

6. Bargen JA, Austin LT (1937) Decalcification of teeth as a result of obstipation with long continued vomiting. Report of a case. J Am Dent Assoc 2: 1271.

7. Christensen GJ (2002) Oral care for patients with bulimia. J Am Dent Assoc 133: 16891691. [Crossref]

8. Mandel L, Kaynar A (1992) Bulimia and parotid swelling: a review and case report. $J$ Oral Maxillofac Surg 50: 1122-1125. [Crossref]

9. Nickl NJ, Brazer SR, Rockwell K, Smith JW (1996) Patterns of esophageal motility in patients with stable bulimia. Am J Gastroenterol 91: 2544-2547. [Crossref]

10. Kamal N, Chami T, Andersen A, Rosell FA, Schuster MM, et al. (1991) Delayed gastrointestinal transit times in anorexia nervosa and bulimia nervosa. Gastroenterology 101: 1320-1324. [Crossref]

11. Koch KL, Bingaman S, Tan L, Stern RM (1998) Visceral perceptions and gastric myoelectrical activity in healthy women and in patients with bulimia nervosa. Neurogastroenterol Motil 10: 3-10. [Crossref]

12. Bosello O, Mazzetti M (2000) Complicanze Mediche dei disturbi del comportamento alimentare: $115-121$

13. Humpries LL, Adams LJ, Eckfeld JH, Levitt MD, Mc Lain CJ (1987) Lexintong, Kentucky; and Minneapolis, Minnesota Hiperamylasemia in patient with eating disorders. Annali of Internal Medicine 106: 50-52.

14. Marano AR, Sangree MH (1984) Acute pancreatitis associated with bulimia. J Clin Gastroenterol 6: 245-248. [Crossref] 
15. Mira M, Stewart PM, Gebski V, Llewellyn-Jones D, Abraham SF (1984) Changes in sodium and uric acid concentrations in plasma during the menstrual cycle. Clin Chem 30: 380-381. [Crossref]

16. Muller E, Brambilla F (1997) Disordini del Comportamento Alimentare: 223-227.

17. Iaffe AC, McAiley LG, Singer L (1987) Carotenemia in normal-weight bulimia: a finding unrelated to other physical manifestations of the syndrome. IntJ Eating Dis 6: 749-755

18. Rissanen A, Naukkarinen H, Virkkunen M, Rawlings RR, Linnoila M(1998) Fluoxetine normalizes increased cardiac vagal tone in bulimia nervosa. J Clin Psychopharmacol 18: 26-32. [Crossref]

19. Meyers DG, Starke H, Pearson PH, Wilken MK (1986) Mitral valve prolapse in anorexia nervosa. Ann Intern Med 105: 384-386. [Crossref]

20. Swenne I (2000) Heart risk associated with weight loss in anorexia nervosa and eating disorders: electrocardiographic changes during the early phase of refeeding. Act Paediatr 89: 447-452. [Crossref]

21. Suri R, Poist ES, Hager WD, Gross JB (1999) Unrecognized bulimia nervosa: a potential cause of perioperative cardiac dysrhythmias. Can J Anaesth 46: 1048-1052. [Crossref]

22. Gupta MA, Gupta AK, Haberman HF (1987) Dermatologic signs in anorexia nervosa and bulimia nervosa. Arch Dermatol 123: 1386-1390. [Crossref]
23. Glorio R, Allevato M, De Pablo A, Abbruzzese M, Carmona L, et al. (2000) Prevalence of cutaneous manifestations in 200 patients with eating disorders. Int J Dermatol 39: 348-353. [Crossref]

24. Bonnetblanc JM, Bonafé JL, Vidal E (1987) Dietary carotenodermias. Ann Dermatol Venereol 114: 1093-1096. [Crossref]

25. Devuyst O, Lambert M, Rodhain J, Lefebvre C, Coche E (1993) Haematological changes and infectious complications in anorexia nervosa: a case control study. $Q \mathrm{~J}$ Med 86: 791-99. [Crossref]

26. Birkmayer NJO, Charlesworth DC, Hernandez F, Leavitt BJ, Marrin CAS, et al. (1998) Obesity and risk of adverse outcomes associated with coronary artery bypass surgery. Circulation 97: 1689-1694. [Crossref]

27. Oster JR, Materson BJ, Rogers AI (1980) Laxative abuse syndrome. Am J Gastroenterol 74: 451-458. [Crossref]

28. Milano W, De Rosa M, Milano L, Riccio A, Sanseverino B, et al. (2013) The pharmacological options in the treatment of eating disorders. ISRN Pharmacol 2013: 352865. [Crossref]

29. Capasso A, Petrella C, Milano W (2009) Pharmacological Profile of SSRIs and SNRIs in the Treatment of Eating Disorders. Curr Clin Pharmacol 4: 78-83. [Crossref]

30. Capasso A, Putrella C, Milano W (2009) Recent clinical aspects of eating disorders Rev Recent Clin Trials 4: 63-69. [Crossref]

Copyright: $(02018$ Milano W. This is an open-access article distributed under the terms of the Creative Commons Attribution License, which permits unrestricted use, distribution, and reproduction in any medium, provided the original author and source are credited. 